\title{
Recent Advances in the Management of Patients with Relapsed/Refractory Follicular Lymphoma
}

\author{
Georgios Pongas (D) \\ Bruce Cheson (1D ${ }^{2}$ \\ 'Department of Medicine, Division of \\ Hematology, Sylvester Comprehensive \\ Cancer Center, University of Miami, \\ Miller School of Medicine, Miami, FL, \\ USA; ${ }^{2}$ Lymphoma Research Foundation, \\ North Bethesda, MD, USA
}

\begin{abstract}
Advanced follicular lymphoma (FL) often relapses after front-line chemoimmunotherapy, and many patients will eventually require subsequent therapy. In 2021, two new therapies were granted approval by the Food and Drug Administration (FDA), including the $\mathrm{PI} 3 \mathrm{~K} \delta$ inhibitor umbralisib and the chimeric antigen receptor-T-cell therapy (CAR-T) axicabtagene ciloleucel. Herein, we present the latest advances in the management of FL, discussing the recently approved therapies in the relapsed and refractory $(R / R)$ setting and various new therapeutic modalities that have the potential to change the treatment landscape and natural history of R/R FL.
\end{abstract}

Keywords: bispecific antibodies, BsAbs, chimeric antigen receptor - T cell therapies, CAR-T, lenalidomide

\section{Introduction}

Follicular lymphoma (FL) is the most common indolent lymphoma in the Western world. ${ }^{1}$ Whilst limited FL is potentially curable with radiation therapy in approximately half of the cases, advanced disease often relapses following front-line chemoimmunotherapy, with many patients requiring repeated forms of treatment. ${ }^{2}$ Herein we present the latest advances in the management of advanced FL and discuss the evolving role of new therapeutic modalities.

\section{Current Approaches for Front-Line Therapy of Advanced Follicular Lymphoma}

Most patients with FL present with advanced-stage disease at diagnosis. In asymptomatic patients with low tumor burden, early intervention with either chemotherapy or rituximab fails to provide a survival advantage, and, therefore, delaying treatment and managing the disease with observation is recommended. ${ }^{3-5}$ The Groupe d'Etude des Lymphomes Folliculaires (GELF) criteria, which were developed to assess tumor burden, are used as a guide to initiate therapy. ${ }^{3}$

For patients with advanced-stage and symptomatic FL, the alkylating agent bendamustine in combination with rituximab $(\mathrm{BR})$ remains the regimen of choice. Its efficacy over R-CHOP was demonstrated in a phase III trial from the Study group indolent Lymphomas (StiL), where the FL patients treated with BR achieved significantly longer progression-free survival (PFS) and superior complete responses (CR). ${ }^{6}$ Subsequently, the phase III BRIGHT study confirmed a superior 5-year PFS of $65.5 \%$ in the BR cohort, compared to $55.8 \%$ in the R-CHOP/R-CVP cohort, and met the primary endpoint demonstrating noninferiority of BR over
Correspondence: Bruce Cheson

Email bdcheson@gmail.com 
R-CHOP as assessed by the CR rates. ${ }^{7,8}$ Whilst the BRIGHT study did not exactly replicate the results of the StiL trial, both studies suggested that BR is a superior chemotherapeutic platform over R-CHOP or R-CVP. ${ }^{7,8}$ Nevertheless, the $65.5 \% 5$-year PFS of BR supports the need for improved therapies. ${ }^{7}$ While maintenance rituximab prolongs the time to disease progression, there is no improvement in survival despite increased toxicity and expense. ${ }^{9}$ One approach to improve on patient outcome has been the development of the nextgeneration anti-CD20 monoclonal antibodies. The one demonstrating the greatest benefit has been obinutuzumab, a glycoengineered, humanized monoclonal anti-CD20 antibody with more potent antibody-dependent cellular cytotoxicity, antibody-dependent phagocytosis and direct cell death compared with rituximab. ${ }^{10}$ The efficacy and safety of obinutuzumab combined with chemotherapy was compared to rituximab-based chemotherapy in the phase III GALLIUM study. ${ }^{11}$ Obinutuzumab with chemotherapy followed by obinutuzumab maintenance achieved a 3-year PFS of $80 \%$ compared to $73.3 \%$ in the rituximabchemotherapy with maintenance rituximab arm, albeit with no prolongation in OS and with more high-grade adverse effects, especially in the bendamustineobinutuzumab arm. ${ }^{11}$ Therefore, because of its increased toxicity, the decision to use bendamustine-obinutuzumab over BR for treatment-naive patients should be carefully balanced.

Whilst chemoimmunotherapy remains the most common treatment for front-line FL, chemotherapy-free options exist. The immunomodulatory combination of lenalidomide with rituximab $\left(\mathrm{R}^{2}\right)$ was evaluated in the phase III study RELEVANCE and was compared with rituximab plus chemotherapy. The primary end points of the study were CR at 120 weeks and PFS, with the CR rate of $\mathrm{R}^{2}$ being $48 \%$ with 3 -year PFS of $77 \%$, similar to the $\mathrm{CR}$ and PFS of immunochemotherapy 53\% and 78\%, respectively. ${ }^{12}$ The ORR to $\mathrm{R}^{2}$ was $61 \%$, similar to $65 \%$ with the immunochemotherapy. Notably, a higher percentage of patients in the R-chemotherapy group had grade 3 or 4 neutropenia $(50 \%$ vs $32 \%)$ and febrile neutropenia ( $7 \%$ vs $2 \%$ ), while a higher rate of grade 3 or 4 cutaneous reactions was observed in the $\mathrm{R}^{2}$ group ( $1 \%$ vs $7 \%$ ). Despite the comparable clinical efficacy and improved safety profile of $\mathrm{R}^{2}$ over chemotherapy, the study was deemed to be negative because it was designed as a superiority trial. Regardless though, the RELEVANCE study demonstrated that immunomodulatory regimens are feasible for treatment-naive FL, paving the way for a new era of chemotherapy-free regimens in the front-line setting of FL. Attempts to improve on the efficacy of $\mathrm{R}^{2}$ have been unsuccessful on the basis of activity and toxicity. ${ }^{13,14}$

\section{Relapsed and Refractory Follicular Lymphoma}

In recent years, multiple effective options have become available for patients with relapsed or refractory FL. The decision of which therapy to choose should be based on response to prior therapies, age, current performance status, comorbidities, goals of therapy and more importantly the safety and efficacy of the treatment.

For rituximab-refractory patients, combined chemoimmunotherapy with a different anti-CD20 monoclonal antibody remains a viable option. In the phase III GADOLIN study, FL patients refractory to rituximab were randomized between bendamustine monotherapy (B) at $120 \mathrm{mg} / \mathrm{m}^{2}$ or obinutuzumab and bendamustine (G-B) at $90 \mathrm{mg} / \mathrm{m}^{2}$ followed by 2 years of obinutuzumab maintenance for those not progressing to G-B. The G-B arm achieved a superior median PFS (mPFS) of 25.8 months compared to 14.1 months of bendamustine monotherapy and also demonstrated a survival benefit. ${ }^{15}$ Notably, $77.5 \%$ of the patients in the G-B arm were refractory to rituximab and an alkylator agent, demonstrating that G-B can have activity in chemotherapy-resistant patients as well. However, given the frequent use of BR in the front-line therapy of FL, it is not clear whether the G-B combination will advance further in the refractory setting.

\section{Chemotherapy-Free Treatment Strategies}

The potential for chemotherapy-free regimens in relapsed and refractory (R/R) FL started with rituximab monotherapy. In a multicenter phase II study, rituximab achieved a $48 \%$ ORR with $6 \% \mathrm{CR}$ and 13 months' median time to progression. ${ }^{16}$ A phase III study from the Swiss Group for Clinical Cancer Research (SAKK) in 202 patients with relapsed or refractory and previously untreated follicular lymphoma showed that 4 weekly doses of rituximab followed by prolonged therapy every 2 months for 4 times increased the event-free survival and response duration compared with the standard weekly $\times 4$ schedule., ${ }^{9,17}$

To enhance the activity of rituximab, the Cancer and Leukemia Group B (CALGB; Alliance) conducted CALGB 50,401, a randomized phase II study comparing lenalidomide alone and $\mathrm{R}^{2}$ in relapsed FL. ${ }^{18}$ The ORR was 
$76 \%$ for $\mathrm{R}^{2}$ with $39 \%$ of patients achieving a CR establishing $\mathrm{R}^{2}$ as a promising combination in relapsed FL. Further support for the role of $\mathrm{R}^{2}$ was provided by the subsequent phase III AUGMENT trial comparing $\mathrm{R}^{2}$ with rituximab plus placebo. Median PFS was significantly longer for the $\mathrm{R}^{2}$ arm at 39.4 months compared to 14.1 months in the rituximab-placebo arm with also longer estimated 2-year OS of $95 \%$ over $86 \%$, respectively. ${ }^{19}$ Similarly, the ORR was superior among patients in the $\mathrm{R}^{2}$ arm $78 \%$ versus $53 \%$, with $34 \%$ versus $18 \%$ achieving CR. Overall, the results of the AUGMENT trial established $\mathrm{R}^{2}$ as a preferred option for $\mathrm{R} / \mathrm{R}$ FL patients who are not refractory to rituximab.

A plethora of new therapeutic modalities since then have demonstrated impressive results in the $R / R$ setting, slowly skewing the treatment of FL away from the traditional chemotherapies. Therapies such as intracellular pathway inhibitors, epigenetic inhibitors and cellular therapies have received approval by the Federal Drug Administration (FDA) and are widely used in the clinical practice (Table 1). New immunotherapies, discussed below, have the potential to change the treatment landscape and natural history of R/R FL.

\section{Monoclonal Antibodies Against Surface Antigens}

Beside the impressive clinical activity of rituximab and obinutuzumab in the front-line and the R/R setting, monoclonal antibodies (mAbs) against other surface antigens have also been evaluated in FL. Galiximab, a chimeric anti-CD80 monoclonal antibody, was combined in a phase II CALGB trial with rituximab and demonstrated $72.1 \%$ ORR with
$47.6 \% \mathrm{CR}$ and mPFS of 2.9 years. ${ }^{20}$ Similarly, epratuzumab, an anti-CD22 monoclonal antibody, when combined with rituximab in a phase II study in treatment-naive FL showed 88.2\% ORR with $42.4 \% \mathrm{CR}$ and a 3-year disease-free survival (DFS) comparable to the survival outcomes and responses to standard chemoimmunotherapies. ${ }^{21}$ These mAbs doublets were the first attempt of combining biological agents and demonstrated an impressive clinical efficacy, but, unfortunately, no regulatory approval was received by the FDA.

Tafasitamab is a humanized anti-CD19 monoclonal antibody which was recently approved by the FDA in combination with lenalidomide for the management of $\mathrm{R} / \mathrm{R}$ diffuse large B-cell lymphoma (DLBCL) not otherwise specified, including DLBCL arising from low-grade lymphoma. Tafasitamab has also demonstrated impressive activity as single agent in R/R FL with $29 \%$ ORR, $6 \% \mathrm{CR}$ and $\mathrm{mDoR}$ of 24 months (Table 2). ${ }^{22}$ The phase III study NCT04680052 is currently assessing the efficacy and safety of tafasitamab in combination with $\mathrm{R}^{2}$ in $\mathrm{R} / \mathrm{R}$ FL compared to placebo plus $\mathrm{R}^{2}{ }^{23}$ If this $\mathrm{mAbs}$ doublet in combination with lenalidomide demonstrates superiority over $R^{2}$, it will replace $R^{2}$ as the new standard of care for second-line therapy of FL.

\section{Antibody Drug Conjugates (ADCs)}

ADCs are highly targeted biopharmaceutical drugs that link a monoclonal antibody against a specific surface antigen to an antitumor cytotoxic molecule. The toxin is thus delivered only to the cells that express the surface antigen, conferring high tumor specificity with limited systemic exposure.

Polatuzumab vedotin is an antibody drug conjugate comprising a humanized anti-CD79B monoclonal antibody

Table I Chemotherapy-Free Regimens Approved for Treatment of Treatment-Naive and Relapsed and Refractory Follicular Lymphoma

\begin{tabular}{|c|c|c|c|c|c|c|}
\hline Drugs & Targets & Combination & Approval Status & ORR/CR & mPFS & Phase \\
\hline \multirow[t]{2}{*}{ Lenalidomide } & \multirow[t]{2}{*}{ Ubiquitin E3 ligase cereblon } & Rituximab & Ist line-upfront & $65 \% / 55 \%$ & NR & $1 I^{12}$ \\
\hline & & Rituximab & 2nd line-Relapsed/Refractory & $79 \% / 32 \%$ & $39.4 \mathrm{mo}$ & $1 I^{19}$ \\
\hline Axi-cel & CAR-T-cell therapy against CDI9 & - & 3rd line- Relapsed/Refractory & $94 \% / 80 \%$ & NR & $11^{64}$ \\
\hline Idelalisib & $\mathrm{PI} 3 \mathrm{~K} \delta$ & - & 3rd line- Relapsed/Refractory & $56 \% / \mid 4 \%$ & II mo & $11^{3 !}$ \\
\hline Duvelisib & $\mathrm{PI} 3 \mathrm{~K} \gamma, \delta$ & - & 3rd line- Relapsed/Refractory & $47 \% / 1 \%$ & $9.5 \mathrm{mo}$ & $11^{36}$ \\
\hline Copanlisib & $\mathrm{PI} 3 \mathrm{~K} \alpha, \delta$ & - & 3rd line- Relapsed/Refractory & $59 \% / 14 \%$ & $11.2 \mathrm{mo}$ & $11^{37}$ \\
\hline Umbralisib & $\mathrm{PI} 3 \mathrm{~K} \delta, \mathrm{CKI} \varepsilon$ & - & 4th line- Relapsed/Refractory & $45 \% / 5 \%$ & $10.6 \mathrm{mo}$ & $1 I^{4 I}$ \\
\hline \multirow[t]{2}{*}{ Tazemetostat } & $\mathrm{EZH} 2^{\text {mut }}$ & - & 3rd line- Relapsed/Refractory & $69 \% / 13 \%$ & $13.8 \mathrm{mo}$ & $11^{55}$ \\
\hline & $\mathrm{EZH} 2^{\mathrm{WT}}$ & - & 3rd line- Relapsed/Refractory & $35 \% / 4 \%$ & II.I mo & $1^{55}$ \\
\hline
\end{tabular}

Abbreviations: ORR, objective response rates; CR, complete response; mPFS, median progression-free survival; mo, months. 
Table 2 Selected Drugs in Development for Follicular Lymphoma

\begin{tabular}{|l|l|l|l|l|l|}
\hline Drugs & Targets & ORR/CR & mPFS & mDoR & Phase \\
\hline Tafasitamab & Anti-CDI9 mAb & $29.4 \% / 5.9 \%$ & $6.6 \mathrm{mo}$ & $24 \mathrm{mo}$ & $11 \mathrm{a}^{22}$ \\
\hline Loncastuximab tesirine & Anti-CDI9 ADC & $78.6 \% / 64.3 \%$ & $\mathrm{NR}$ & $\mathrm{NR}$ & $\mathrm{I}^{27}$ \\
\hline Odronextamab & CD20 $\times$ CD3 BsAb & $92.9 \% / 75 \%(\geq 5 \mathrm{mg})$ & $12.8 \mathrm{mo}$ & $7.7 \mathrm{mo}$ & $\mathrm{I}^{58}$ \\
\hline Mosunetuzumab & CD20 $\times$ CD3 BsAb & $68 \% / 50 \%$ & $11.8 \mathrm{mo}$ & $20.4 \mathrm{mo}$ & $1 / 1 \mathrm{l}^{61}$ \\
\hline Epcoritamab & CD20 $\times$ CD3 BsAb & $100 \% / 25 \%(\geq 0.76 \mathrm{mg})$ & $\mathrm{NR}$ & $\mathrm{NR}$ & $1 / 1 \mathrm{I}^{62}$ \\
\hline Glofitamab & CD20 $\times$ CD3 BsAb & $70.5 \% / 47.7 \%$ & $11.8 \mathrm{mo}$ & $10.8 \mathrm{mo}$ & $1^{63}$ \\
\hline
\end{tabular}

Abbreviations: ORR, objective response rates; $\mathrm{CR}$, complete response; $\mathrm{mPFS}$, median progression-free survival; mDoR, median duration of response; $\mathrm{mAb}$, monoclonal antibody; mo, months; ADC, antibody drug conjugate; BsAb, bispecific antibody.

conjugated to the microtubule-disrupting monomethyl auristatin E. Polatuzumab vedotin in combination with rituximab was evaluated in the phase II clinical trial ROMULUS in R/R non-Hodgkin lymphoma (NHL), where it demonstrated a $70 \%$ ORR among 20 patients with FL, with $45 \%$ of patients achieving a CR. ${ }^{24}$ The median PFS was 15.4 months with a median DoR of 9.4 months. The combination was well tolerated with most common TEAEs being fatigue, diarrhea, peripheral neuropathy, nausea and neutropenia. Polatuzumab vedotin has also been combined with obinutuzumab in a phase $\mathrm{Ib} / \mathrm{II}$ study in R/R NHL where it achieved a 78\% ORR in patients with FL. ${ }^{25}$ While the polatuzumab appears to have some clinical activity in R/R FL, its FDA approval is currently restricted for the therapy of $\mathrm{R} / \mathrm{R}$ DLBCL in combination with bendamustine and rituximab due to the absence of clinical benefit from the addition of polatuzumab to BR in FL. ${ }^{26}$

Loncastuximab tesirine consists of a humanized anti-CD19 monoclonal antibody conjugated to a pyrrolobenzodiazepine dimer toxin. Loncastuximab tesirine was evaluated in a phase I study in R/R NHL, where it demonstrated a $78.6 \%$ ORR in FL. ${ }^{27}$ Most common TEAEs were hematologic AEs including thrombocytopenia, neutropenia and anemia, whereas fatigue, elevation of the gamma-glutamyltransferase, peripheral edema and pleural effusions were the most common non-hematological TEAEs. Based on the promising activity and the acceptable safety profile, more combinations of loncastuximab tesirine are currently in development. Given the recent FDA priority review of loncastuximab tesirine for $\mathrm{R} / \mathrm{R}$ DLBCL, a plausible approval for $\mathrm{R} / \mathrm{R} \mathrm{FL}$ may follow if loncastuximab proves to be superior to idelalisib in the ongoing phase II LOTIS 6 clinical trial in R/R FL (NCT04699461).

\section{Drugs That Interfere with the B-Cell Receptor Signaling: PI3K and BTK Inhibitors PI3K Inhibitors}

The role of B-cell receptor (BCR) signaling in the maintenance and progression of $\mathrm{FL}$ is well characterized. ${ }^{28}$ The PI3K pathway is downstream from the BCR and is vital for the survival of FL. There are four class I PI3K isoforms in mammals, which are named after the p110 catalytic subunits. ${ }^{29}$ The $p 110 \alpha$ and $p 110 \beta$ are characterized by ubiquitous tissue distribution, while the $\mathrm{p} 110 \gamma$ and $\mathrm{p} 110 \delta$ are mainly expressed in the hematopoietic system including B, T and NK cells. Notably, p110 $\delta$ is downstream from the BCR and along with the $\mathrm{p} 110 \alpha$ are important for B-cell development. Currently four PI3K inhibitors are approved for R/R FL: idelalisib, duvelisib and copanlisib for thirdline therapy, and umbralisib approved for fourth-line.

Idelalisib is an oral $\mathrm{PI} 3 \mathrm{~K} \delta$ inhibitor that was evaluated in a phase II study in patients with indolent $\mathrm{R} / \mathrm{R}$ NHL, where $56 \%$ of the FL patients responded and $14 \%$ achieved a CR. ${ }^{30,31}$ Responses in FL were rapid and durable with median time to response (mTTR) of 2.6 months, median duration of response (mDoR) of 10.8 months and mPFS of 11 months. Most frequent grade 3 or greater AEs included elevation of aminotransferase levels and diarrhea in $13 \%$ and pneumonia in $7 \% .{ }^{30}$ The median follow-up of this study was only 6 months, and many of the immune-mediated adverse effects occur later during treatment. While longer follow-up of up to 6.7 years did not reveal any new safety issues and indicated beneficial outcome, major concerns limiting its use related to 5 black box warnings from the FDA for fatal and/or severe diarrhea or colitis, hepatotoxicity, 
pneumonitis and intestinal perforation. ${ }^{32}$ More serious unexpected toxicities were also noted in the combination studies of idelalisib. ${ }^{33}$ Grade 3 or higher pneumonitis was observed in $17 \%$ of the patients in a phase II study combining the SYK inhibitor entospletinib with idelalisib leading to two fatalities. ${ }^{34}$ Similarly, two phase I studies of idelalisib with $\mathrm{R}^{2}$ had to be terminated due to excessive unexpected toxicities such as severe transaminitis, septic shock, hypotension with rash, and lung infection. ${ }^{35}$

Duvelisib, a first-in-class oral dual $\mathrm{PI} 3 \mathrm{~K} \gamma, \delta$ inhibitor was evaluated in a phase II study in patients with indolent NHL (iNHL) and demonstrated $42.2 \%$ ORR with only $1 \%$ $\mathrm{CR}$ in $\mathrm{R} / \mathrm{R} \mathrm{FL}$, which appears lower than other drugs in that class. ${ }^{36}$ Among the most frequent AEs were diarrhea (48.8\%) and cough (27.1\%). The most frequent grade 3 or greater AEs were neutropenia (24.8\%) and diarrhea $(14.7 \%)$, whereas colitis and pneumonitis were reported in $7.8 \%$ and $4.7 \%$, respectively. ${ }^{36}$ The drug has 4 black box warnings, including diarrhea/colitis and pneumonitis along with cutaneous reactions and infections.

Copanlisib is a pan-class I PI3K inhibitor with predominant activity against $\mathrm{PI} 3 \mathrm{~K} \alpha$ and $\mathrm{PI} 3 \mathrm{~K} \delta$, which is administered at $60 \mathrm{mg}$ IV on days 1,8 and 15 of a 28 -day cycle. Copanlisib was evaluated in a phase II study in patients with indolent R/R NHL. ${ }^{37}$ Among 104 patients with FL, the ORR was $59 \%$ with $15 \%$ achieving a CR. The responses were rapid and durable with $\mathrm{mDoR} 12.2$ months and mPFS of 11.2 months. ${ }^{37}$ The most frequent TEAEs occurring in $\geq 25 \%$ of patients were transient hyperglycemia, presumably related to targeting the alpha isoform, transient hypertension, diarrhea, fatigue, decreased neutrophil count and fever. Notably the incidence of severe GI toxicities such as hepatic transaminitis, colitis, diarrhea and colonic perforation were less common with the intermittent intravenous copanlisib compared with the prior reports of idelalisib and duvelisib, and therefore no black box warning accompanies copanlisib. ${ }^{38}$ Additionally, copanlisib was the first PI3K inhibitor to be safely combined with rituximab based on the CHRONOS-III study, where it demonstrated superior PFS of 21.5 months versus 13.8 months with rituximab and placebo. ${ }^{39} \mathrm{~A}$ possible explanation for the more favorable profile may be the intermittent dosing schedule which has been designed to achieve optimal target inhibition within the tumor while sparing the normal tissue and has proven to be more effective than the continuous administration in animal models. Currently, copanlisib is being evaluated in the phase III clinical trials CHRONOS-II and IV (NCT02369016, NCT02626455) in R/R iNHL as monotherapy or in combination with traditional chemoimmunotherapy BR or R-CHOP, respectively, and the results are eagerly anticipated. ${ }^{38,40}$

Umbralisib is an oral, first-in-class, dual inhibitor of $\mathrm{PI} 3 \mathrm{~K} \delta$ and casein kinase- $1 \varepsilon(\mathrm{CK} 1 \varepsilon)$. CK1 $\varepsilon$ is a key component of the noncanonical Wnt signaling pathway which has been shown to drive the pathogenesis of B-cell lymphoproliferative disorders. In the phase IIb UNITY study, 208 patients with $\mathrm{R} / \mathrm{R}$ indolent lymphoma were administered umbralisib $800 \mathrm{mg}$ orally once daily. ${ }^{41}$ With a median follow-up of 27.7 months among 117 patients with FL, the ORR was $45.3 \%$ with $5.1 \%$ achieving a CR. The median TTR was 4.6 months with a median PFS of 10.6 months and a median DoR of 11.1 months. The responses were similarly impressive in marginal zone lymphoma (MZL) and SLL with $49.3 \%$ and $50 \%$ ORR and $15.9 \%$ and $4.5 \% \mathrm{CR}$, respectively. The most common grade 3 or greater AEs were neutropenia, diarrhea (10\%) and increased ALT/AST (6.7\%/7.2\%). Notably, pneumonitis and noninfectious colitis were only observed at small frequency of $1.4 \%$ and $1.9 \%$, respectively, suggesting a favorable benefit-risk profile. The precise reason for the toxicities observed with the first-generation PI3K inhibitors is not yet known, albeit there are indications that the regulatory T-cells (Treg) number and function may be preserved in umbralisib-treated patients, which could partially explain the improved safety profile. ${ }^{42}$ Whist umbralisib appears to have a better safety profile compared with idelalisib and duvelisib, its regulatory approval for fourthline therapy in R/R FL stems from the fact that the median number of prior systemic therapies in the UNITY study was 3 and that there was no unmet medical need with the $\mathrm{R}^{2}$ in second-line and tazemetostat and the other PI3K inhibitors for the third-line.

Additional PI3K inhibitors are currently under clinical development such as the next-generation $\mathrm{PI} 3 \mathrm{~K} \delta$ inhibitors parsaclisib and zandelisib. In a phase II study in patients with R/R FL, parsaclisib demonstrated an ORR of $69.8 \%$ with $13.5 \% \mathrm{CR} .^{43}$ The mTTR and the mPFS were 8 weeks and 15.8 months, respectively, whereas the most common grade $\geq 3$ TEAEs were diarrhea $(9.4 \%)$, neutropenia (6.6\%) and colitis $(3.8 \%)$. In the phase II trial, continuous vs intermittent dosing schedules of zandelisib are currently evaluated in patients with $\mathrm{R} / \mathrm{R}$ FL to evaluate the riskbenefit profile of these two treatment schedules. ${ }^{44}$ 
Overall, these studies demonstrate that PI3K inhibition can have a clinically meaningful outcome in FL. Nevertheless, autoimmune related toxicities such as pneumonitis and colitis remain a challenge that may interfere with the compliance to the therapy and can lead to discontinuation. The newer-generation PI3K inhibitors, copanlisib and umbralisib, appear to be better tolerated, albeit larger-scale studies and real-world data are required to fully understand their tolerability and position among the other two PI3K inhibitors. Ongoing clinical studies are evaluating the role of new-generation PI3K $\delta$ inhibitors and may expand further the PI3K $\delta$ armamentarium. Combinations of PI3K $\delta$ should be carefully designed to avoid reproducing the disastrous outcomes of prior studies. Avoiding combinations with SYK inhibitors or immunomodulatory drugs and exploring combinations with the newer CD19 monoclonal antibodies such as loncastuximab or tafasitamab may lead to better tolerated therapies with improved efficacy.

\section{BTK Inhibitors}

The role of BTK inhibitors in FL appears to be modest based on the low clinical activity in the phase II studies. ${ }^{45,46}$ In the phase II consortium trial of ibrutinib in R/R FL, ibrutinib demonstrated an ORR of $37.5 \%$ with $12.5 \%$ CR and median PFS of 14 months. ${ }^{46}$ The response rates were significantly higher among patients sensitive to rituximab (52.6\%) compared with those who were rituximab-refractory (16.7\%). Notably, CARD11 mutations, which are known to confer resistance to ibrutinib, were present in $16 \%$ of patients, and only the CARD $11^{\mathrm{WT}}$ responded. However, the DAWN study, which also assessed the efficacy of ibrutinib in R/R FL, failed to meet its primary point demonstrating an ORR of $20.9 \%{ }^{45}$ Nevertheless, the median DoR was 19.4 months with $11 \%$ of patients achieving a CR. Correlative studies showed a significant downregulation of $\mathrm{CD} 4{ }^{+} \mathrm{CD} 25^{+} \mathrm{CD} 127^{-}$regulatory T-cells (Tregs) in the responders but not in nonresponders along with increase in Th1-promoting (antitumor) cytokines interferon- $\gamma$ and interleukin-12. Based on these results, it is unlikely for ibrutinib monotherapy to advance further in the management of FL, and hence novel combinations with other therapeutics are currently under clinical development. Additionally, a new generation of BTK inhibitors such as zanubrutinib are currently undergoing clinical trials which may show more meaningful outcomes. ${ }^{47}$

\section{BCL2 Inhibitors: Venetoclax}

Venetoclax (VEN) is a highly selective BCL-2 inhibitor with important activity in CLL as a single agent and in combinations with anti-CD20s and ibrutinib. As a single agent it has also demonstrated activity against 5 different subtypes of R/R non-Hodgkin lymphoma (NHL) with an ORR of $38 \%$ with $14 \% \mathrm{CR}$ for FL and an estimated mPFS of 11 months. ${ }^{48}$ The subsequent phase Ib study CAVALLI combined VEN with rituximab (arm A) or obinutuzumab (arm B) and CHOP in patients with FL and DLBCL. ${ }^{49}$ Across both treatment arms, the ORR was $83.3 \%$ in $\mathrm{FL}$ with $75 \%$ of patients achieving a CR and a 1 -year PFS of $100 \%$ for R-CHOP and $90 \%$ for G-CHOP. The most common AEs in both arms were neutropenia and nausea, with cytopenias being predominant among grade $3 / 4$ events and were reported more frequently in the G-CHOP arm. In a phase IB study of VEN in combination with $\mathrm{BR}$ in $\mathrm{R} / \mathrm{R}$ NHL, the ORR was $75 \%$ for the FL with $25 \%$ of the patients achieving a $\mathrm{CR}$, which does not appear to be superior to the historical ORR and CR of BR in R/R FL. ${ }^{50}$ Since the $\mathrm{mPFS}$ and $\mathrm{mDoR}$ were not reached for the FL patients, additional follow-up is needed to better understand whether this combination will have a durable response. Similarly, the phase II CONTRALTO study which assessed the safety and efficacy of VEN+rituximab, and VEN+BR vs BR alone in $\mathrm{R} / \mathrm{R}$ FL, the combined VEN+BR achieved similar efficacy with the BR with ORR of $75 \%$ vs $69 \%$, respectively, albeit with higher toxicity leading to lower dose intensity of BR. ${ }^{51}$ Based on these two studies, it is unlikely that the addition of VEN to BR will move forward to clinic.

Combinations of venetoclax with tyrosine kinase inhibitors have also been explored. In the first phase I, chemotherapy-free combination of venetoclax with the BTK inhibitor ibrutinib in R/R FL, there was a ORR of $69 \%$ with $25 \%$ of patients achieving a CR and a median PFS of 8.3 months. ${ }^{52}$ There was no evidence of clinical tumor lysis syndrome (TLS), and the most common grade 3 AEs were neutropenia and thrombocytopenia. These results were encouraging, and further evaluation at the dose of $560 \mathrm{mg}$ ibrutinib and $600 \mathrm{mg}$ venetoclax is ongoing in a phase II trial. The combination of ibrutinib with venetoclax may eventually provide another alternative chemotherapy-free option for R/R FL.

\section{Epigenetic Therapies: EZH2 Inhibitor-Tazemetostat}

Enhancer of zeste homolog 2 (EZH2) histone methyltransferase is required for the germinal center formation, and it is mutated in $25 \%$ of FL. ${ }^{53,54}$ Tazemetostat is an oral, first-inclass inhibitor of the mutant and wild type (WT) EZH2. 
Tazemetostat was evaluated in a phase II study in 99 patients with R/R FL and demonstrated $69 \%$ ORR in the EZH2 ${ }^{\text {mut }}$ compared to $35 \%$ in the EZH $2{ }^{\mathrm{WT}}$ cohort, with median PFS of 13.8 months versus 11.1 months, respectively. ${ }^{55}$ It is important to note, however, that there were some important differences of the baseline patient characteristics between the two cohorts. Specifically, only $24 \%$ of the $\mathrm{EZH} 2^{\text {mut }}$ cohort had relapsed after receiving a $\mathrm{PI} 3 \mathrm{~K}$ inhibitor or an immunomodulatory drug, compared to $39 \%$ in the $\mathrm{EZH} 2{ }^{\mathrm{WT}}$ group. Also, the $\mathrm{EZH} 2{ }^{\mathrm{WT}}$ group was more heavily pretreated with a median of 3 lines of prior anticancer therapy compared to 2 for the $\mathrm{EZH} 2^{\text {mut }}$ cohort. Additionally, the $\mathrm{EZH} 2^{\mathrm{WT}}$ was characterized by higher rates of other poor risk features such as prior hematopoietic stem-cell transplant and POD24 in $39 \%$ and $59 \%$, respectively, compared to $9 \%$ and $42 \%$ in the $\mathrm{EZH} 2^{\text {mut }}$ cohort. It unclear whether these imbalances in the baseline patient characteristics represent a higher vulnerability of $\mathrm{EZH} 2{ }^{\text {mut }} \mathrm{FL}$ to the available therapies, a more aggressive phenotype of the EZH $2^{\mathrm{WT}} \mathrm{FL}$, or there was a selection bias. Nevertheless, the outcome results led to the FDA approval of tazemetostat for the therapy of patients with $\mathrm{R}$ R FL whose tumors are positive for the EZH2 mutation and who have received at least two prior systemic therapies and also for patients with R/R FL who have no satisfactory alternative treatment options. ${ }^{56}$

A systemic literature review showed that tazemetostat has a favorable safety profile. Based on its safety and efficacy, tazemetostat may be prioritized over the PI3K inhibitors for third-line use in patients with EZH2 mutations. Combinations of tazemetostat with other therapeutics such as rituximab and lenalidomide are currently under investigation and will hopefully improve the survival outcomes compared to the single agent tazemetostat. ${ }^{57}$

\section{Immunotherapies: Bispecific Antibodies, Chimeric Antigen Receptor-T-Cell Therapies and Macrophage Check Point Inhibitors Bispecific Antibodies}

Bispecific antibodies (BsAbs) are designed to bind to different epitopes on various cell types. Therefore, BsAbs can improve the tumor eradication by bringing the cytotoxic T-cells or natural killer (NK)-cells in closer proximity to the tumor cells. Those BsAbs currently undergoing clinical development in FL include the CD20 x CD3s odronextamab, mosunetuzumab, epcoritamab and glofitamab.
Odronextamab (REGN1979) is a first-in-class, hingestabilized, intravenously administered, fully human IgG4based $\mathrm{BsAb}$ that binds to CD20-expressing B cells and CD3 on T-cells, which can engage both targets inducing T-cell activation and cytotoxicity. In a phase I study of odronextamab in R/R NHL, $92.9 \%$ ORR was noted at doses $\geq 5 \mathrm{mg}$ in patients with FL, with $75 \%$ achieving a $\mathrm{CR}^{58}$ The median duration of complete response (DoCR) was 8.1 months with mPFS of 12.8 months, and follow-up is ongoing. Most common TEAEs among the overall population of 127 patients were pyrexia, chills and cytokine release syndrome (CRS) with median duration of 2 days; $11 \%$ of the CRS was grade 3, and the severity of the CRS declined through optimized premedication. ${ }^{59}$ A phase II multicohort study was recently designed to assess the antitumor activity and safety of odronextamab in patients with B-NHL, where 112 patients are estimated to have FL. ${ }^{60}$

Mosunetuzumab is an intravenously administered, fully humanized immunoglobulin G1 CD20/CD3 BsAb and is currently evaluated in a phase $\mathrm{I} / \mathrm{Ib}$ trial in R/R NHL. ${ }^{61}$ Among 62 patients with FL, mosunetuzumab achieved a $68 \%$ ORR with $50 \%$ CR. Notably, consistent CR rates were observed in those with double refractory disease (55\%), disease progression before 24 months (POD24) (53\%), refractory to PI3K inhibition (78\%) and those who had received prior CAR-T therapy $(50 \%)$. The mDoR was 20.4 months, whereas the mPFS was 11.8 months. Most common grade 3 or higher AEs were hypophosphatemia and neutropenia. While $23 \%$ of patients experienced CRS, only $1.6 \%$ of the CRS was classified as a serious AE (SAE). Overall, mosunetuzumab was deemed to have high response rates and resulted in durable disease control.

Epcoritamab is a subcutaneously administered BsAb that simultaneously binds to $\mathrm{CD} 3$ on T-cells and CD20 on $\mathrm{B}$ cells and is currently evaluated in a phase I/II trial in patients with R/R NHL. ${ }^{62}$ Among 8 patients with FL who received epcoritamab $\geq 0.76 \mathrm{mg}, 100 \%$ achieved an ORR with at least $25 \%$ CR. Notably, PET CT was not used for disease assessment in all patients with PR, and therefore it is plausible that the CR rates may have been higher. While pyrexia, fatigue and injection site reactions were the most common TEAEs, the CRS observed with higher doses were all grade 1-2. These results suggest a favorable safety profile which could support the outpatient administration of epcoritamab.

Glofitamab is a novel intravenously administered BsAb that has a longer half-life compared with non-Fc-bearing 
bispecific T-cell engagers. In a phase I study among 171 patients with R/R B-NHL, glofitamab achieved 70.5\% ORR in 44 patients with FL, with $47.7 \% \mathrm{CR}^{63}$ While the $\mathrm{mDoR}$ among the 31 responders was 10.8 months, $90.5 \%$ of patients with $\mathrm{CR}$ remained in $\mathrm{CR}$ up to 22.9 months, demonstrating that the $\mathrm{CR}$ can be long-lived. Notably the CRS was manageable, with low rates of grade $\geq 3$ and no treatment withdrawals.

Overall, the BsAbs have shown promising activity in heavily pretreated patients with FL. A main advantage over the CAR-T is their availability as off-the-shelf products, unlike the autologous cell processing with the lengthy periods of genetic engineering and expansion. However, whether BsAbs will demonstrate long-lived DoR like the CAR-T remains uncertain, and a longer follow-up period is required. A potential long-term durable remission in combination the impressive ORR may eventually change the natural history and treatment landscape of R/R FL. Following the evaluation of BsAbs in the R/R setting with such impressive response rates, clinical trials in the upfront setting should also be considered. Given the reliance of BsAbs to the endogenous T-cells, it seems tempting to speculate that their use in treatment-naive patients with more robust T-cell fitness may demonstrate even greater complete responses. Additionally, various combinations of BsAbs with other immunotherapies should be also explored. Merging immunotherapies that can simultaneously boost the innate and adaptive immunity such as combining anti-CD47 mAbs with BsAbs may lead to improved complete responses with more prolonged durations.

\section{Chimeric Antigen Receptor-T-Cell Therapies}

Chimeric antigen receptor (CAR) T-cell therapies against CD19 have revolutionized the treatment of relapsed and refractory DLBCL and acute lymphoblastic leukemia (ALL). Based on these results, CAR-T have been explored in largely incurable lymphomas including FL. More recently the FDA approved the use of axicabtagene ciloleucel (axi-cel) based on results of the ZUMA-5 phase II study for adult patients with R/R FL after two or more lines of systemic therapy.

Axi-cel is an autologous anti-CD19 chimeric antigen receptor (CAR) T-cell therapy, which is approved for the treatment of R/R DLBCL. In the ZUMA-5 study, among 84 patients with $R / R$ FL after $\geq 2$ lines of therapy, the ORR to axi-cel was $94 \%$, with $80 \%$ achieving a $\mathrm{CR}^{6}{ }^{64}$ The 12 -month DoR, PFS and OS among the overall population were $72 \%$, $74 \%$ and $93 \%$, respectively. Whilst grade 3 AEs or greater occurred in $85 \%$ of the patients with FL, grade 3 or greater CRS and neurologic events occurred in $6 \%$ and $15 \%$ of FL, respectively. Interestingly, patients were eligible for retreatment if they progressed after achieving a response at a 3-month post infusion assessment. ${ }^{65}$ Among 11 retreated patients, 9 with FL and 2 with marginal zone lymphoma (MZL), all responded with $91 \%$ achieving a CR and with a median follow-up of 2.3 months; the median DoR was not achieved. Similarly, a phase I/II study evaluating CD19-CAR -T in R/R FL demonstrated an $88 \%$ CR rate among 8 patients. The median time to CR was 29 days, and all patients who achieved a $\mathrm{CR}$ remained in remission for a median follow-up of 24 months, demonstrating a long-lasting effect. ${ }^{66}$ Notably no severe (grade $\geq 3$ ) CRS or neurotoxicity was observed.

In summary, based on the activity of the CAR-T with its durable clinical benefit, this approach has the potential to change the natural history of FL. Whether there will be a clear winner among different CAR-T products remains to be seen in future clinical trials. Main challenges remain, including the management of the CRS and the neurologic events. Possible intervention with agents to mitigate the toxicity or new CAR-T products is an area of evolving investigation, but overall the risk and benefit of CAR-T favor their use in FL.

\section{Macrophage Check Point Inhibitors: Anti-CD47 Antibodies}

CD47 is a transmembrane protein which is present on normal cells but overexpressed in cancer cells and functions as a "don't eat me" signal, allowing cells to evade the immune-mediated eradication by silencing the macrophages through binding of SIRP $\alpha$, resulting in inhibition of phagocytosis. ${ }^{67-69}$ Antibodies directed against CD47 are immune check point inhibitors that can induce an antitumor response through phagocytosis of the tumor cell by the macrophage and also by induction of antitumor T-cell response through cross-presentation of tumor antigens by phagocytes to T-cells.

Magrolimab is a first-in-class anti-CD47 monoclonal antibody. In a phase IB study in patients with R/R NHL, the combination of magrolimab with rituximab demonstrated a $71 \%$ ORR with $43 \%$ CR among 7 patients with FL, previously refractory to rituximab regimens. ${ }^{70}$ At a median follow-up of 8.1 months, $91 \%$ of the responses 
were ongoing. Most common AEs included anemia and IRS and were predominantly grade 1-2.

Additional CD47 checkpoint pathway inhibitors such as TTI622 and ALX148 are also undergoing clinical development, and the updated outcome data will provide more insight about the position of the macrophage checkpoint inhibitors in the management of R/R FL. ${ }^{71,72}$

\section{T-Cell Check Point Inhibitors: Anti-Programmed Death-I Antibodies}

Nivolumab is a fully human IgG4 mAb against the programmed death-1 (PD-1), which releases the inhibition of the T-cells and restores the antitumor immune responses. Based on some promising activity of nivolumab in the phase I study CheckMate 039, where an ORR of $40 \%$ was observed in $\mathrm{R} / \mathrm{R}$ FL, the phase II trial CheckMate 140 assessed nivolumab in 92 patients with R/R FL. ${ }^{73,74}$ Unfortunately, nivolumab showed very limited activity with a ORR of $4 \%$ and a mPFS of 2.2 months, demonstrating that single-agent PD-1 blockade is not effective in $R / R$ FL. At this point, the role of the PD-1 inhibitors in the therapeutic arena of FL remains unclear.

\section{Conclusions}

A number of novel approaches have the potential to improve the natural history of patients with FL. Impressive response rates have been reported with BsAbs and CAR-T-cell therapy in the relapsed and refractory setting, and future clinical trials should investigate the efficacy of BsAbs in the first-line setting. BsAbs appear more appealing as off-the-shelf products especially for symptomatic patients who cannot wait for the T-cell manufacturing and processing. Perhaps priority should be given to the elderly patients that are deemed not fit for chemotherapy and then advance to the younger population, should the outcome data look promising. In the meantime, while chemoimmunotherapy remains the standard of care for initial treatment, its role in the relapsed and refractory setting is clearly vanishing, and the front-line will soon follow suit. Lenalidomide with rituximab is the combination of choice for second-line therapy, although a role for tafasitamab is being explored in these rituximab-failed patients. The clinical efficacy and safety of tazemetostat in the thirdline was impressive, questioning how it would compare with $\mathrm{R}^{2}$, but instead it is being combined with $\mathrm{R}^{2}$ in a phase III registration study. In regard to the treatment of choice in the third-line, axi-cel will likely be prioritized over the PI3K inhibitors in select patients given its superior response rates and durability of responses. Among the PI3K inhibitors, it appears that umbralisib is similarly active and the best tolerated with less auto-immune related toxicities and, therefore, may eventually dominate, especially when approved in earlier lines of therapy. Importantly, outcome data of BsAbs and CAR--T-cell therapy are maturing and will provide information on the durability of their benefit. In the meanwhile, thoughtful and rationally designed clinical trials utilizing a chemo-free approach with thorough correlative studies and identification of biomarkers to individualize therapy are needed to transform this mostly incurable disease to a readily curable lymphoma.

\section{Author Contributions}

All authors made a significant contribution to the work reported, whether that is in the conception, study design, execution, acquisition of data, analysis and interpretation, or in all these areas; took part in drafting, revising or critically reviewing the article; gave final approval of the version to be published; have agreed on the journal to which the article has been submitted; and agree to be accountable for all aspects of the work.

\section{Disclosure}

GP has received honoraria from Curio Science and OncLive and has served on advisory board for Atara Biotherapeutics. BC received personal fees for consulting and/or member of advisory boards from Celgene, Morphosys, Kite, Epizyme, Beigene, Symbios, Lilly, and TG Therapeutics, personal fees from AbbVie, Gilead, Karyopharm, Merck, Glaxo Smith Kline, and Janssen/ Pharmacyclics, during the conduct of the study. The authors report no other potential conflicts of interest for this work.

\section{References}

1. Teras LR, DeSantis CE, Cerhan JR, Morton LM, Jemal A, Flowers CR. 2016 US lymphoid malignancy statistics by World Health Organization subtypes. CA Cancer J Clin. 2016;66 (6):443-459. doi:10.3322/caac.21357

2. Lo AC, Campbell BA, Pickles T, et al. Long-term outcomes for patients with limited-stage follicular lymphoma: update of a population-based study. Blood. 2020;136(8):1006-1010. doi:10.1182/ blood.2019004588

3. Brice P, Bastion Y, Lepage E, et al. Comparison in low-tumor-burden follicular lymphomas between an initial no-treatment policy, prednimustine, or interferon alfa: a randomized study from the Groupe d'Etude des lymphomes folliculaires. Groupe d'Etude des Lymphomes de l'Adulte. J Clin Oncol. 1997;15(3):1110-1117. doi:10.1200/JCO.1997.15.3.1110 
4. Ardeshna KM, Smith P, Norton A, et al. Long-term effect of a watch and wait policy versus immediate systemic treatment for asymptomatic advanced-stage non-hodgkin lymphoma: a randomised controlled trial. Lancet. 2003;362(9383):516-522. doi:10.1016/S0140-6736(03)14110-4

5. Ardeshna KM, Qian W, Smith P, et al. Rituximab versus a watch-andwait approach in patients with advanced-stage, asymptomatic, non-bulky follicular lymphoma: an open-label randomised phase 3 trial. Lancet Oncol. 2014;15(4):424-435. doi:10.1016/S14702045(14)70027-0

6. Rummel MJ, Niederle N, Maschmeyer G, et al. Bendamustine plus rituximab versus CHOP plus rituximab as first-line treatment for patients with indolent and mantle-cell lymphomas: an open-label, multicentre, randomised, phase 3 non-inferiority trial. Lancet. 2013;381(9873):1203-1210. doi:10.1016/S0140-6736(12)61763-2

7. Flinn IW, van der Jagt R, Kahl B, et al. First-line treatment of patients with indolent non-hodgkin lymphoma or mantle-cell lymphoma with bendamustine plus rituximab versus R-CHOP or R-CVP: results of the BRIGHT 5-year follow-up study. J Clin Oncol. 2019;37 (12):984-991. doi:10.1200/JCO.18.00605

8. Flinn IW, van der Jagt R, Kahl BS, et al. Randomized trial of bendamustine-rituximab or R-CHOP/R-CVP in first-line treatment of indolent NHL or MCL: the BRIGHT study. - PubMed - NCBI. Blood. 2014;123(19):2944-2952. doi:10.1182/blood-2013-11-531327

9. Moccia AA, Taverna C, Schär S, et al. Prolonged rituximab maintenance in follicular lymphoma patients: long-term results of the SAKK 35/03 randomized trial. Blood Adv. 2020;4(23):5951-5957. doi:10.1182/bloodadvances. 2020002858

10. Freeman CL, Sehn LH. A tale of two antibodies: obinutuzumab versus rituximab. Br J Haematol. 2018;182(1):29-45.

11. Marcus R, Davies A, Ando K, et al. Obinutuzumab for the first-line treatment of follicular lymphoma. $N$ Engl J Med. 2017;377 (14):1331-1344. doi:10.1056/NEJMoa1614598

12. Morschhauser F, Fowler NH, Feugier P, et al. Rituximab plus lenalidomide in advanced untreated follicular lymphoma. $N$ Engl J Med. 2018;379(10):934-947. doi:10.1056/NEJMoa1805104

13. Ujjani C, Wang H, Skarbnik A, et al. A phase 1 study of lenalidomide and ibrutinib in combination with rituximab in relapsed and refractory CLL. Blood Adv. 2018;2(7):762-768. doi:10.1182/ bloodadvances.2017015263

14. Smith SM, Pitcher BN, Jung SH, et al. Safety and tolerability of idelalisib, lenalidomide, and rituximab in relapsed and refractory lymphoma: the alliance for clinical trials in oncology A051201 and A051202 phase 1 trials. Lancet Haematol. 2017;4(4):e176-e182. doi:10.1016/S2352-3026(17)30028-5

15. Cheson BD, Chua N, Mayer J, et al. Overall survival benefit in patients with rituximab-refractory indolent non-hodgkin lymphoma who received obinutuzumab plus bendamustine induction and obinutuzumab maintenance in the GADOLIN Study. J Clin Oncol. 2018;36 (22):2259-2266. doi:10.1200/JCO.2017.76.3656

16. McLaughlin P, Grillo-Lopez AJ, Link BK, et al. Rituximab chimeric anti-CD20 monoclonal antibody therapy for relapsed indolent lymphoma: half of patients respond to a four-dose treatment program. J Clin Oncol. 1998;16(8):2825-2833. doi:10.1200/JCO.1998.16.8.2825

17. Ghielmini M, Schmitz SF, Cogliatti SB, et al. Prolonged treatment with rituximab in patients with follicular lymphoma significantly increases event-free survival and response duration compared with the standard weekly x 4 schedule. Blood. 2004;103(12):4416-4423. doi:10.1182/blood-2003-10-3411

18. Leonard JP, Jung SH, Johnson J, et al. Randomized trial of lenalidomide alone versus lenalidomide plus rituximab in patients with recurrent follicular lymphoma: CALGB 50401 (Alliance). J Clin Oncol. 2015;33(31):3635-3640. doi:10.1200/JCO.2014.59.9258

19. Leonard JP, Trneny M, Izutsu K, et al. AUGMENT: a Phase III Study of lenalidomide plus rituximab versus placebo plus rituximab in relapsed or refractory indolent lymphoma. J Clin Oncol. 2019;37 (14):1188-1199. doi:10.1200/JCO.19.00010
20. Czuczman MS, Leonard JP, Jung S, et al. Phase II trial of galiximab (anti-CD80 monoclonal antibody) plus rituximab (CALGB 50402): Follicular Lymphoma International Prognostic Index (FLIPI) score is predictive of upfront immunotherapy responsiveness. Ann Oncol. 2012;23(9):2356-2362. doi:10.1093/annonc/mdr620

21. Grant BW, Jung SH, Johnson JL, et al. A phase 2 trial of extended induction epratuzumab and rituximab for previously untreated follicular lymphoma: CALGB 50701. Cancer. 2013;119(21):3797-3804. doi:10.1002/cncr.28299

22. Jurczak W, Zinzani PL, Hess G, et al. A Phase IIa, Open-Label, Multicenter Study of single-agent tafasitamab (MOR208), an Fcoptimized anti-CD19 antibody, in patients with relapsed or refractory B-cell non-hodgkin's lymphoma: long-term follow-up, final analysis. Blood. 2019;134(Supplement_1):4078. doi:10.1182/blood-2019-124297

23. A Phase 3 Study to assess efficacy and safety of tafasitamab plus lenalidomide and rituximab compared to placebo plus lenalidomide and rituximab in patients with Relapsed/Refractory (R/R) follicular Lymphoma or Marginal Zone Lymphoma. (InMIND). Available from: https://clinicaltrials.gov/ct2/show/NCT04680052?term= NCT04680052\&draw=2\&rank=1. Accessed July 14, 2021.

24. Morschhauser F, Flinn IW, Advani R, et al. Polatuzumab vedotin or pinatuzumab vedotin plus rituximab in patients with relapsed or refractory non-hodgkin lymphoma: final results from a phase 2 randomised study (ROMULUS). Lancet Haematol. 2019;6(5):e254e265. doi:10.1016/S2352-3026(19)30026-2

25. Phillips T, Brunvand M, Chen A, et al. Polatuzumab vedotin combined with obinutuzumab for patients with relapsed or refractory non-hodgkin lymphoma: preliminary safety and clinical activity of a Phase Ib/II Study. Blood. 2016;128(22):622. doi:10.1182/blood. V128.22.622.622

26. Sehn LH, Kamdar M, Herrera AF, et al. Randomized phase 2 trial of polatuzumab vedotin (pola) with bendamustine and rituximab (BR) in relapsed/refractory (r/r) FL and DLBCL. J Clin Oncol. 2018;36 (15_suppl):7507. doi:10.1200/JCO.2018.36.15_suppl.7507

27. Hamadani M, Radford J, Carlo-Stella C, et al. Final results of a Phase 1 Study of loncastuximab tesirine in relapsed/refractory B-cell non-hodgkin lymphoma. Blood. 2021;137(19):2634-2645.

28. Kuppers R. Mechanisms of B-cell lymphoma pathogenesis. Nat Rev Cancer. 2005;5(4):251-262. doi:10.1038/nrc1589

29. Pongas G, Cheson BD. PI3K signaling pathway in normal B cells and indolent B-cell malignancies. Semin Oncol. 2016;43(6):647-654. doi:10.1053/j.seminoncol.2016.11.011

30. Gopal AK, Kahl BS, de Vos S, et al. PI3Kdelta inhibition by idelalisib in patients with relapsed indolent lymphoma. $N$ Engl J Med. 2014;370(11):1008-1018. doi:10.1056/NEJMoa1314583

31. Salles G, Schuster SJ, de Vos S, et al. Efficacy and safety of idelalisib in patients with relapsed, rituximab- and alkylating agent-refractory follicular lymphoma: a subgroup analysis of a phase 2 study. Haematologica. 2017;102(4):e156-e159. doi:10.3324/haematol.20 16.151738

32. Wagner-Johnston ND, Schuster SJ, deVos S, et al. Outcomes of patients with up to 6 years of follow-up from a phase 2 study of idelalisib for relapsed indolent lymphomas. Leuk Lymphoma. 2021;62 (5):1077-1087.

33. Cheson BD. Speed bumps on the road to a chemotherapy-free world for lymphoma patients. Blood. 2016;128(3):325-330. doi:10.1182/ blood-2016-04-709477

34. Barr PM, Saylors GB, Spurgeon SE, et al. Phase 2 study of idelalisib and entospletinib: pneumonitis limits combination therapy in relapsed refractory CLL and NHL. Blood. 2016;127(20):2411-2415. doi:10.1182/blood-2015-12-683516

35. Smith SM, Pitcher B, Jung S-H, et al. Unexpected and serious toxicity observed with combined idelalisib, lenalidomide and rituximab in relapsed/refractory B cell lymphomas: alliance A051201 and A051202. Blood. 2014;124(21):3091. doi:10.1182/blood.V124.21. 3091.3091 
36. Flinn IW, Miller CB, Ardeshna KM, et al. DYNAMO: a phase II Study of duvelisib (IPI-145) in patients with refractory indolent non-hodgkin lymphoma. J Clin Oncol. 2019;37(11):912-922. doi:10.1200/JCO.18.00915

37. Dreyling M, Santoro A, Mollica L, et al. Phosphatidylinositol 3-kinase inhibition by copanlisib in relapsed or refractory indolent lymphoma. J Clin Oncol. 2017;35(35):3898-3905. doi:10.1200/ JCO.2017.75.4648

38. Chauhan AF, Cheson BD. Copanlisib in the treatment of relapsed follicular lymphoma: utility and experience from the clinic. Cancer Manag Res. 2021;13:677-692. doi:10.2147/CMAR.S201024

39. Matasar MJ, Capra M, Özcan M, et al. Copanlisib plus rituximab versus placebo plus rituximab in patients with relapsed indolent non-Hodgkin lymphoma (CHRONOS-3): a double-blind, randomised, placebo-controlled, phase 3 trial. Lancet Oncol. 2021;22 (5):678-689.

40. Nowakowski GS, Gorbatchevsky I, Hiemeyer F, Cupit L, Childs BH. Abstract CT212: CHRONOS-2: a randomized, double-blind phase III study of phosphatidylinositol-3 kinase alpha/delta inhibitor copanlisib versus placebo in patients with rituximab-refractory indolent nonHodgkin's lymphoma (iNHL). Cancer Res. 2015;75(15Supplement): CT212-CT212.

41. Fowler NH, Samaniego F, Jurczak W, et al. Umbralisib, a dual $\mathrm{PI} 3 \mathrm{Kdelta} / \mathrm{CK} 1 \mathrm{epsilon}$ inhibitor in patients with relapsed or refractory indolent lymphoma. J Clin Oncol. 2021;39:JCO2003433.

42. Maharaj K, Powers JJ, Achille A, et al. The dual PI3Kdelta/ CK1 epsilon inhibitor umbralisib exhibits unique immunomodulatory effects on CLL T cells. Blood Adv. 2020;4(13):3072-3084. doi:10.1182/bloodadvances.2020001800

43. Lynch RC, Paneesha S, Avigdor A, et al. Phase 2 Study evaluating the efficacy and safety of parsaclisib in patients with relapsed or refractory follicular lymphoma (CITADEL-203). Blood. 2020;136 (Supplement 1):36-37. doi:10.1182/blood-2020-134869

44. Zelenetz AD, Zinzani PLL, Chan H, et al. ME-401-003 (TIDAL): a multicenter, randomized, double-blind, placebo-controlled, two-arm, Phase 2 Study of ME-401 investigating continuous and intermittent dosing schedules in patients with relapsed/refractory follicular lymphoma. Blood. 2019;134(Supplement_1):5244. doi:10.1182/blood-2019-124326

45. Gopal AK, Schuster SJ, Fowler NH, et al. Ibrutinib as treatment for patients with relapsed/refractory follicular lymphoma: results from the open-label, multicenter, Phase II DAWN Study. J Clin Oncol. 2018;36(23):2405-2412. doi:10.1200/JCO.2017.76.8853

46. Bartlett NL, Costello BA, LaPlant BR, et al. Single-agent ibrutinib in relapsed or refractory follicular lymphoma: a phase 2 consortium trial. Blood. 2018;131(2):182-190. doi:10.1182/blood-201709-804641

47. Fowler NH, Trotman J, Auer R, et al. Randomized Phase 2 zanubrutinib (BGB-3111) + obinutuzumab vs obinutuzumab monotherapy in patients with relapsed/refractory follicular lymphoma (R/R FL). Blood. 2019;134(Supplement_1):5252. doi:10.1182/blood-2019-122628

48. Davids MS, Roberts AW, Seymour JF, et al. Phase I first-in-Human Study of venetoclax in patients with relapsed or refractory non-hodgkin lymphoma. $J$ Clin Oncol. 2017;35(8):826-833. doi:10.1200/JCO.2016.70.4320

49. Zelenetz AD, Salles G, Mason KD, et al. Venetoclax plus R- or G-CHOP in non-hodgkin lymphoma: results from the CAVALLI phase 1b trial. Blood. 2019;133(18):1964-1976. doi:10.1182/blood2018-11-880526

50. de Vos S, Swinnen LJ, Wang D, et al. Venetoclax, bendamustine, and rituximab in patients with relapsed or refractory NHL: a phase $\mathrm{Ib}$ dose-finding study. Ann Oncol. 2018;29(9):1932-1938. doi:10.1093/ annonc/mdy 256

51. Zinzani PL, Flinn IW, Yuen SLS, et al. Venetoclax-rituximab with or without bendamustine vs bendamustine-rituximab in relapsed/refractory follicular lymphoma. Blood. 2020;136(23):2628-2637.
52. Ujjani CS, Lai C, Leslie LA, et al. Ibrutinib and venetoclax in relapsed and refractory follicular lymphoma. Blood. 2020;136 (Supplement 1):46-47. doi:10.1182/blood-2020-136219

53. Beguelin W, Popovic R, Teater M, et al. EZH2 is required for germinal center formation and somatic EZH2 mutations promote lymphoid transformation. Cancer Cell. 2013;23(5):677-692. doi:10.1016/j.ccr.2013.04.011

54. Bodor C, Grossmann V, Popov N, et al. EZH2 mutations are frequent and represent an early event in follicular lymphoma. Blood. 2013;122 (18):3165-3168. doi:10.1182/blood-2013-04-496893

55. Morschhauser F, Tilly H, Chaidos A, et al. Tazemetostat for patients with relapsed or refractory follicular lymphoma: an open-label, single-arm, multicentre, phase 2 trial. Lancet Oncol. 2020;21 (11):1433-1442. doi:10.1016/S1470-2045(20)30441-1

56. FDA Adminstration. Available from: https://www.fda.gov/drugs/fdagranted-accelerated-approval-tazemetostat-follicular-lymphoma. Accessed July 14, 2021.

57. Patel K, Bailey N, Pagel JM. A phase 2, Open-Label, Multicenter Study of tazemetostat in combination with rituximab for the treatment of relapsed or refractory follicular lymphoma. Blood. 2020;136 (Supplement 1):4. doi:10.1182/blood-2020-136653

58. Bannerji R, Allan JN, Arnason JE, et al. Odronextamab (REGN1979), a human CD20 x CD3 bispecific antibody, induces durable, complete responses in patients with highly refractory B-cell non-hodgkin lymphoma, including patients refractory to CAR T therapy. Blood. 2020;136(Supplement 1):42-43. doi:10.1182/blood-2020-136659

59. Bannerji R, Arnason JE, Advani R, et al. Emerging clinical activity of REGN1979, an anti-CD20 $\mathrm{x}$ anti-CD3 bispecific antibody, in patients with relapsed/refractory Follicular Lymphoma (FL), Diffuse Large B-Cell Lymphoma (DLBCL), and other B-Cell Non-Hodgkin Lymphoma (B-NHL) subtypes. Blood. 2018;132(Supplement 1):1690. doi:10.1182/blood-2018-99-113328

60. Kim TM, Alonso A, Prince M, et al. A Phase 2 Study of Odronextamab (REGN1979), a CD20 x CD3 bispecific antibody, in patients with relapsed/refractory B-cell non-hodgkin lymphoma. Blood. 2020;136 (Supplement 1):28-29. doi:10.1182/blood-2020-136344

61. Assouline SE, Kim WS, Sehn LH, et al. Mosunetuzumab shows promising efficacy in patients with multiply relapsed follicular lymphoma: updated clinical experience from a phase I dose-escalation trial. Blood. 2020;136(Supplement 1):42-44. doi:10.1182/blood2020-135839

62. Hutchings M, Mous R, Clausen MR, et al. Subcutaneous epcoritamab induces complete responses with an encouraging safety profile across relapsed/refractory B-cell non-hodgkin lymphoma subtypes, including patients with prior CAR-T therapy: updated dose escalation data. Blood. 2020;136(Supplement 1):45-46. doi:10.1182/blood-2020-133820

63. Hutchings M, Morschhauser F, Iacoboni G, et al. Glofitamab, a novel, bivalent CD20-targeting T-cell-engaging bispecific antibody, induces durable complete remissions in relapsed or refractory B-cell lymphoma: a phase I trial. J Clin Oncol. 2021;JCO:20.03175.

64. Jacobson C, Chavez JC, Sehgal AR, et al. Primary analysis of zuma-5: a phase 2 Study of Axicabtagene Ciloleucel (Axi-Cel) in patients with Relapsed/Refractory (R/R) Indolent Non-Hodgkin Lymphoma (iNHL). Blood. 2020;136(Supplement 1):40-41.

65. Chavez JC, Jacobson CA, Sehgal AR, et al. Retreatment with Axicabtagene Ciloleucel (Axi-Cel) in patients with relapsed/refractory indolent non-hodgkin lymphoma in ZUMA-5. Blood. 2020;136 (Supplement 1):34. doi:10.1182/blood-2020-135871

66. Hirayama AV, Gauthier J, Hay KA, et al. High rate of durable complete remission in follicular lymphoma after CD19 CAR-T cell immunotherapy. Blood. 2019;134(7):636-640. doi:10.1182/blood. 2019000905

67. Chao MP, Weissman IL, Majeti R. The CD47-SIRPalpha pathway in cancer immune evasion and potential therapeutic implications. Curr Opin Immunol. 2012;24(2):225-232. doi:10.1016/j.coi.2012.01.010 
68. Tsai RK, Discher DE. Inhibition of "self" engulfment through deactivation of myosin-II at the phagocytic synapse between human cells. $J$ Cell Biol. 2008;180(5):989-1003. doi:10.1083/jcb.200708043

69. Campbell IG, Freemont PS, Foulkes W, Trowsdale J. An ovarian tumor marker with homology to vaccinia virus contains an IgV-like region and multiple transmembrane domains. Cancer Res. 1992;52 (19):5416-5420.

70. Advani R, Flinn I, Popplewell L, et al. CD47 blockade by Hu5F9-G4 and rituximab in non-hodgkin's lymphoma. N Engl J Med. 2018;379 (18):1711-1721. doi:10.1056/NEJMoa1807315

71. Patel K, Ramchandren R, Maris $M$, et al. Investigational CD47-blocker TTI-622 shows single-agent activity in patients with advanced relapsed or refractory lymphoma: update from the Ongoing First-in-Human Dose Escalation Study. Blood. 2020;136(Supplement 1):46-47. doi:10.1182/blood-2020-136607
72. Kim TM, Lakhani N, Gainor J, et al. ALX148, a CD47 blocker, in combination with rituximab in patients with non-hodgkin lymphoma. Blood. 2020;136(Supplement 1):13-14. doi:10.1182/blood-2020135941

73. Armand P, Janssens A, Gritti G, et al. Efficacy and safety results from CheckMate 140, a phase 2 study of nivolumab for relapsed/refractory follicular lymphoma. Blood. 2021;137(5):637-645. doi:10.1182/ blood.2019004753

74. Lesokhin AM, Ansell SM, Armand P, et al. Nivolumab in patients with relapsed or refractory hematologic malignancy: preliminary results of a Phase Ib Study. J Clin Oncol. 2016;34(23):2698-2704. doi:10.1200/JCO.2015.65.9789

\section{Publish your work in this journal}

Blood and Lymphatic Cancer: Targets and Therapy is an international, peer-reviewed, open access journal focusing on blood and lymphatic cancer research, identification of therapeutic targets and the optimal use of preventative and integrated treatment interventions to achieve improved outcomes, enhanced survival and quality of life for the cancer patient. The manuscript management system is completely online and includes a very quick and fair peer-review system. Visit http://www.dovepress.com/testimonials.php to read real quotes from published authors. 